\title{
Qualitative Assessment of Research done at Two Premier Medical Institutes in India: Some Data for Introspection
}

\author{
Ravi Prakash Kanojia, Kirti Kumar Rathod, KLN Rao
}

\section{ABSTRACT}

Aim: To perform a qualitative assessment of research publications coming from two premier medical institutes in India.

Materials and methods: Research publications from AlIMS and PGIMER published during 2004 to 2008 were analyzed. The publications were categorized into five types. A year-wise analysis was done for both the institutes. Data was obtained from Scopus. The collected data included the number of papers published in a category, total citations received by a type of publication in a year, number of publications which have no citations, number of publications with only one citation, h-index, number of zero and only one citation were added to get publications with negligible citations.

Results: A total number of 4,828 and 2,882 research publications were analyzed from AIIMS and PGIMER respectively. The original articles attracted highest no of citations that is 64 and $66 \%$ of the total citations in a year for AIIMS and PGIMER respectively. Approximately 37 to $42 \%$ of the original articles get negligible citations. The $\mathrm{h}$-index for various categories of article was higher for the original articles (18/13), whereas it was very low for other category of research publications.

Conclusion: The original articles fetch more citations in long run. Close to $40 \%$ of the original research done in the studied institutes get negligible citations pointing toward their outcome utility and quality. More than $60 \%$ of the minor category publications remain uncited. This sort of data if periodically obtained should serve as a benchmark for quality analysis of scientific research.

Keywords: Citation analysis, Bibliometric analysis, Scopus, Scientific research h-index.

How to cite this article: Kanojia RP, Rathod KK, Rao KLN. Qualitative Assessment of Research done at Two Premier Medical Institutes in India: Some Data for Introspection. J Postgrad Med Edu Res 2012;46(1):24-33.

Source of support: Nil

Conflict of interest: None declared

\section{INTRODUCTION}

Scientific research is one of the primary mandates of a tertiary care institute. The benchmark for the magnitude and the quality of research done by a tertiary care hospital is judged by the number of research publications (RP) originating from an institute. Higher is the number of RP coming from a particular center, greater is the research credibility associated with that institute. People and research centers have been emphasizing on this concept of numbers and have made it synonymous with research credibility. With the availability of internet-based bibliometric databases, it has been observed that merely numbers may not be sufficient for qualitative assessment of the research done by a particular institute or an individual. In order to have a better and exact qualitative assessment of a research, we present an analysis using the concept of citations associated with a RP. ${ }^{1}$ A simple methodology is outlined for qualitatively assessing the publications coming from institutes based on Scopus citation analysis (SCA) with analysis being done for All India Institute of Medical Sciences (AIIMS), New Delhi, and Postgraduate Institute of Medical Education and Research (PGIMER), Chandigarh, the two premier medical institutes of India. The data available from such analysis projects the quality of research output from an institute as well as serves as a benchmark for other institutions serving the same mandate.

\section{MATERIALS AND METHODS}

We analyzed the entire publications from AIIMS and PGIMER published between January 2004 and December 2008. The analysis was done in a year-wise manner separately for both the institutes with no data comparison between the two institutes. The publications were divided into six types, namely Original Article (OA), Review Articles (RA), Editorial (Ed), Case Reports (CR), Letter to Ed (LEd) and Miscellaneous (Misc). The OA category grouped publications lying at the highest level of evidence, i.e. the randomized control trials, systemic analysis, meta analysis, original case series where as the miscellaneous category contained various undefined type of publications as brief reports, note, snippet, conference presentations, etc. The data was obtained separately for each category. The no of citations obtained by each category of publication was obtained via Scopus ${ }^{2}$ which is an online service provided by Elsevier. Scopus gives a paid access to such data; we used the authorized institutional login for this purpose. The year limit was kept within the mentioned time. The data collected included the number of papers published in a category in a particular year, total citations received by a type of publication in a year, number of publications which have no citations (zero citations), number of publications with only one (negligible) citations, h-index (this is defined later), number of zero and only one citation were added and were taken as publications with negligible citations. 
The publication trends in various categories were also observed.

The mentioned values for each year were calculated as percentage against the respective variables to give a simplified over view to the reader. Before we present the analysis of the data recorded it would be prudent to define terminology used in the analysis.

h-index: Is an indicative value calculated on the basis of number of citations received either by an individual, periodical, a set of articles or an institution. ${ }^{3}$ The calculation is based on the scientist's most cited papers. This can also be applicable to a group of scientists, a department, university, or a country. The index was suggested by Jorge E Hirsch. ${ }^{4}$ The precise definition given by him is 'number of papers having equal number of citations'. In other words, a scholar with an index of $h$ has published $h$ number of papers each of which has been cited $h$ number of times. The h-index reflects both number of publications and the number of citations per publication. The index value is used to compare scientists working in the same field. The value does not give importance to the number of publications. Hence, for two scientists or institutes one can have greater h-index even if he has lesser number of publications provided his publications are highly cited this will happen only if he has done substantial quality work. The index has been used by some analyst for journals and they give greater importance to it as compared to impact factor. Researchers have been rated at several places and have been promoted based on this value. As mentioned previously, we have grouped the publications from AIIMS and PGIMER into six types and calculated their h-index in a year-wise fashion. This will reflect the overall quality of papers published in that category. It is now clear that higher the value, better is the academic quality of the work or the institute.

\section{RESULTS}

For the clarity of description, the results and observations are given separately for the two institutes.

\section{AllMS}

The total number of published papers from AIIMS between January 2004 and December 2008 was 4828. The various genres with their relative percentage are depicted in Figure 1. It is obvious that medicine is the predominant category to which majority of the publications belong constituting $66 \%$ of the total publications. The year wise as well as the article category wise data is presented in Tables 1 and 2. Observations made on individual category of papers are now described.

\section{Original Articles}

Total number of 2,180 OA were published with an average of 436 per year in the last 5 years. The number of OA published per year has been on an increasing trend. The OA make $45 \%$ of the total published papers each year. These OA get an average of $64 \%$ of the total citations received in that year. Approximately 23\% of the OA published in a year get zero citations and an average of $14 \%$ of OA get only one citation which is negligible. Hence, average $37 \%$ $(23+14)$ of OA published each year get negligible citations. The publication trend seen during the 5 -year period has been toward increasing numbers.

\section{Review Articles}

Total 440 review articles were published in last 5 years with and average of 88 per year which is $9 \%$ of the total articles published in that year. The citation received by these RA averages to $16 \%$ of the total citations received in that year. On an average, $31 \%$ of the RA published in a year do not get any citation. Eleven percent were able to get only one citation which is negligible. Hence, $42 \%(31+11)$ of the RA published in a year get negligible citations.

\section{Editorial}

Total 93 editorials were published in last 5 years with an average of 18 per year which is $1.8 \%$ of the total papers published in respective year. These editorials were able to get $0.6 \%$ of the total citations obtained in respective year. On an average $55 \%$ of the editorials published do not get any citations and $12 \%$ are able to obtain only one citation which is negligible. Hence, $67 \%(55+12)$ of the editorials published each year get negligible citations.

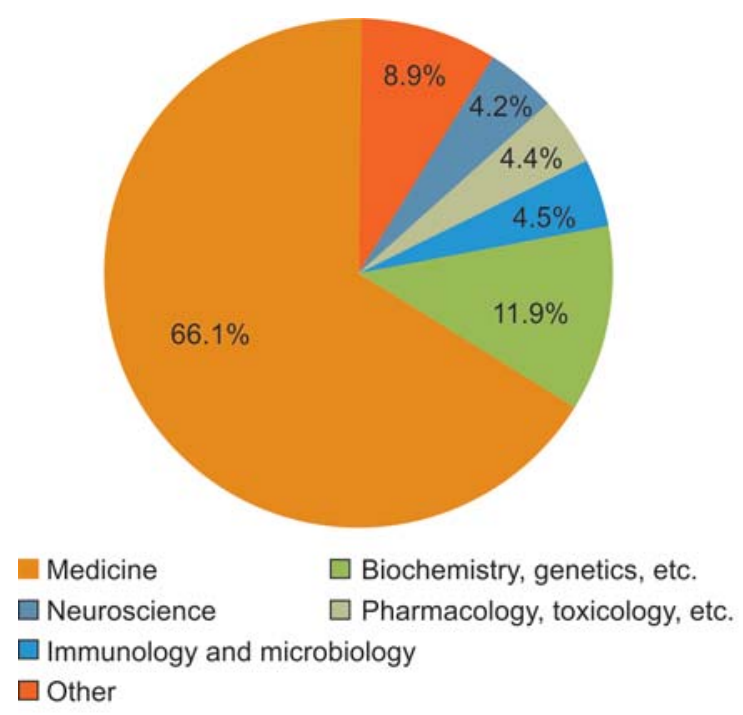

Fig. 1: Various genres of publications from AlIMS with their relative percentage 


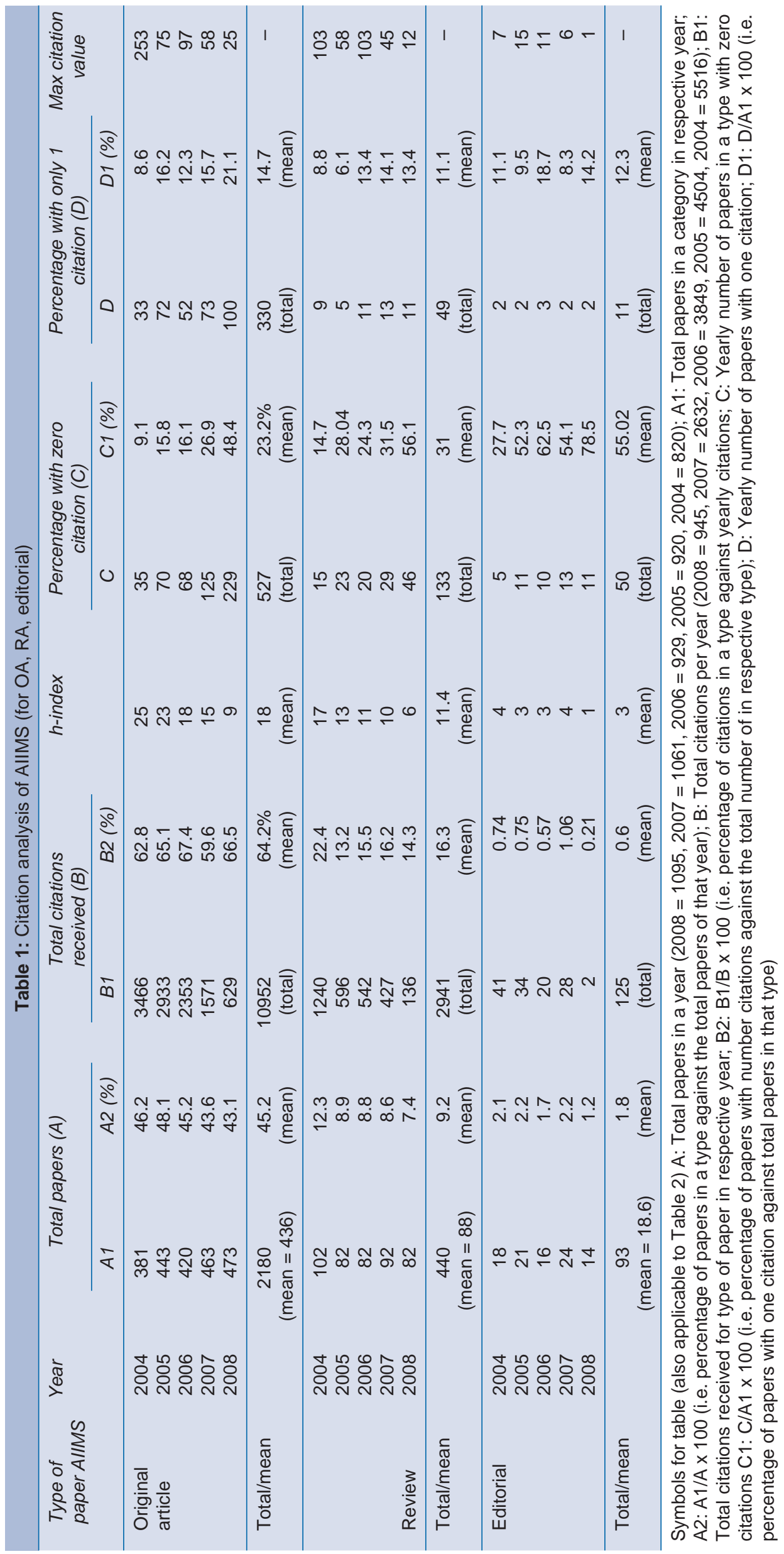




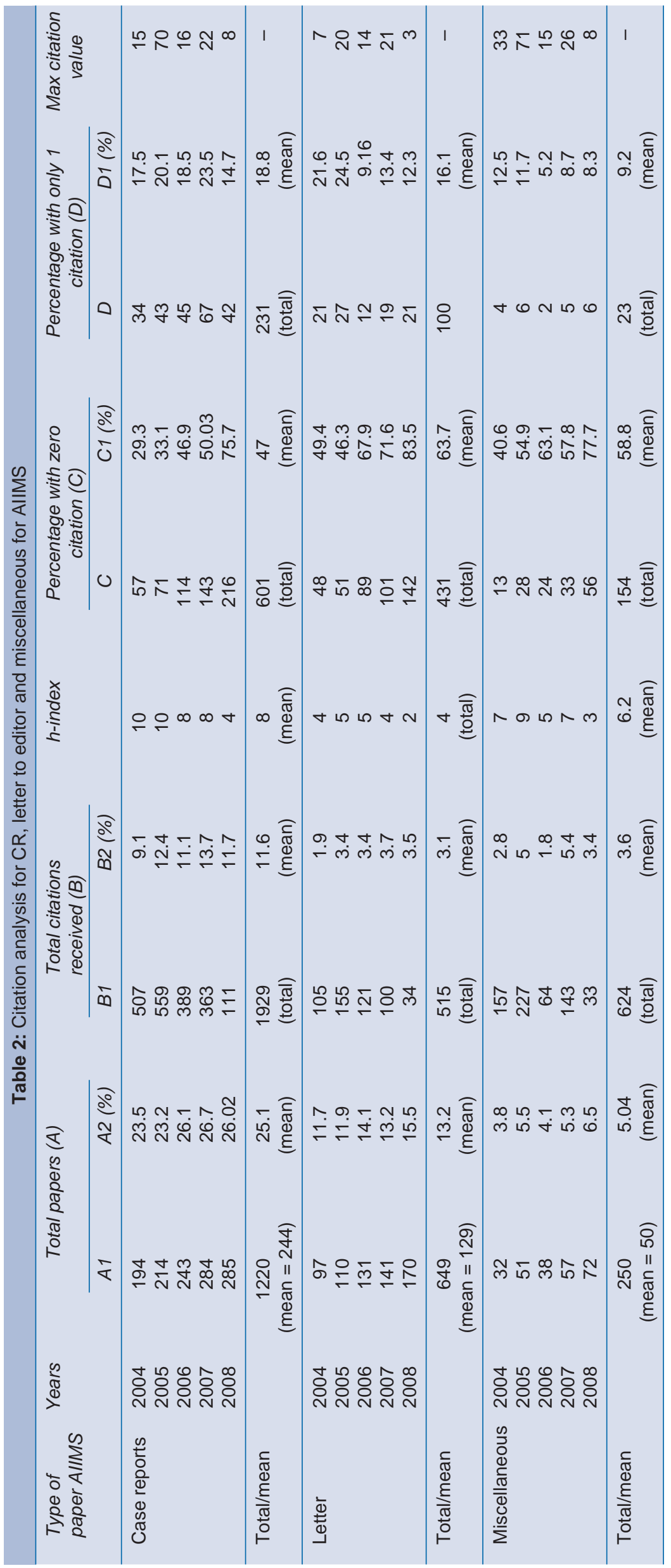




\section{Case Reports}

Total 1224 CR were published in last 5 years with an average of 244 per year which is $25 \%$ of total papers published each year. These CR get an average of $11.6 \%$ of the total citations obtained in respective year. On an average 47\% of the CR do not get any citations and 19\% get only one citation which is negligible. Hence, $66 \%(47+19)$ of the CR published get negligible citations.

\section{Letter to Editor}

Total 649 letters were published with an average of 129 per year. They form average of $13 \%$ of the total papers published in respective year. They are able to fetch $3.1 \%$ of the total citations in a year. On an average $63 \%$ of the let published get no citations at all and $16 \%$ get only one citation which is negligible. Hence, $79 \%(63+16)$ of the letters published each year get negligible citations.

\section{Miscellaneous}

Fifty papers are published in this category each year which fetch $3 \%$ of the total citations for the respective year. Fiftynine percent of these papers do not get any citations and $9 \%$ get only one citations. Hence, $68 \%$ of these papers get negligible citations.

The entire publication numbers against the percentage of papers with negligible citations is presented in the histogram (Fig. 2).

\section{PGIMER}

The total number of published papers were 2882. The various genre with their relative percentage is depicted in Figure 3. It is obvious that medicine is the predominant category to which majority of the publications belong

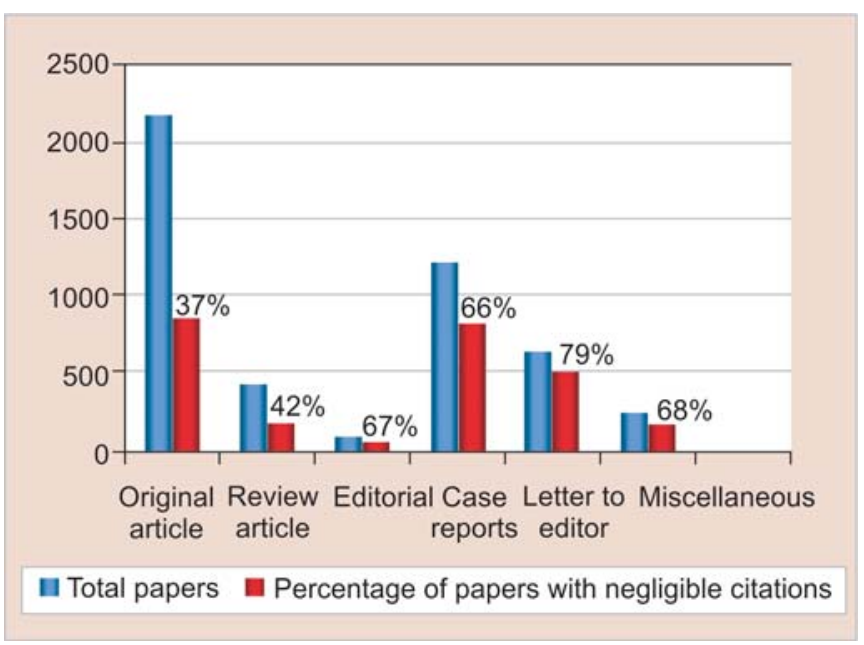

Fig. 2: Publication numbers against the percentage of papers with negligible citations from AlIMS

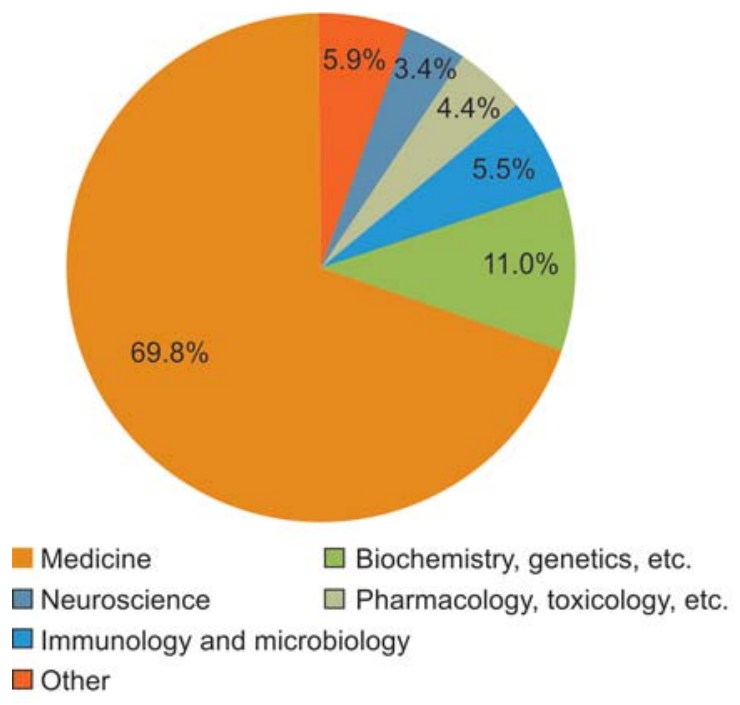

Fig. 3: Various genres of publications from PGIMER with their relative percentage

constituting $70 \%$ of the total publications. The year wise as well as category wise data is presented in Tables 3 and 4 . Observations made on individual category of papers are now described.

\section{Original Articles}

Average number of original articles published per year is 250 which constitutes an average of $43 \%$ of the total articles published every year. Year 2006 had maximum number of OA published in all the 5 years analyzed. The number of citations for OA per year averages to $66 \%$ of the total citations received in that year. On an average, $26 \%$ of the OA fail to get any citations at all. Sixteen percent of the published OA got only one citation which is close to negligible. Hence, $42 \%(16+26)$ of the published OA in a year gets negligible citations.

\section{Review Articles}

On an average $43 \mathrm{RA}$ are published each year which is $7.5 \%$ of the total articles published in that year. The year 2006 had maximum number of RA published in all the 5 years analyzed. The number of citations received per year averages to $12 \%$ of the total citations received for all types of articles published in that year. On an average $27 \%$ of the RA published per year fail to get any citation at all. And $13 \%$ of the RA gets only one citation which is negligible. Hence, $40 \%(27+13)$ of the RA published every year get negligible citations.

\section{Editorials}

Average number of editorials published every year is 8 which is $1.4 \%$ of the total articles published in that year. The number of editorials has doubled during the last 5 years. But the citations received with these editorials has fallen 


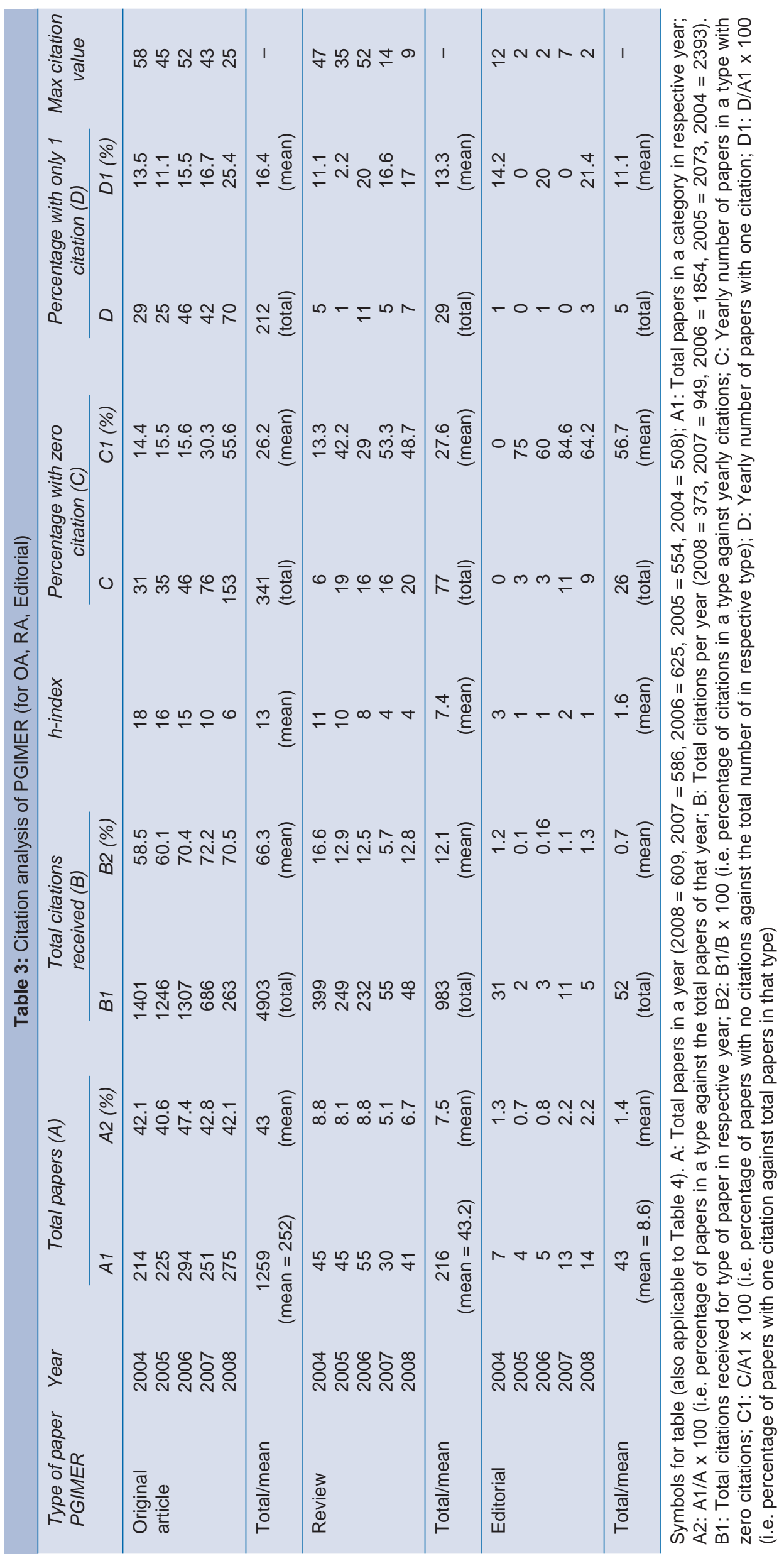




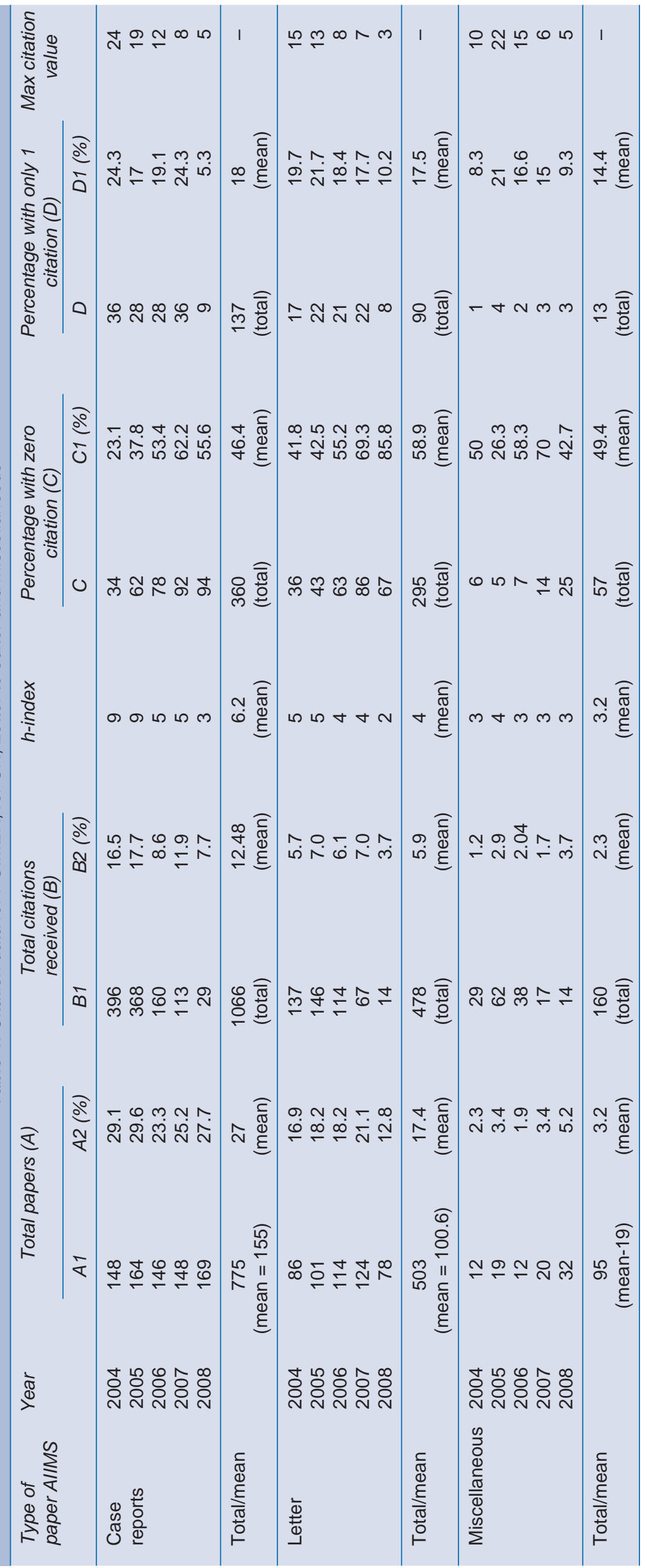


sharply. The average citation received has been 10 per year which is less than $1 \%$ of the total citations received. The range during the 5 years has been 1.3 to $0.1 \%$. On an average $56 \%$ of the editorials do not receive any citations and $11 \%$ per year get only one citations. Hence, $67 \%(56+11)$ of the editorials get negligible citations.

\section{Case Reports}

Average number of CR published per year is 155 which is $27 \%$ of the total articles published in that year. The average citations received by these $\mathrm{CR}$ is averaged at $12 \%$ of the total citations obtained in that year. On an average $46 \%$ of these CR do not get any citations and $18 \%$ get only one citation which is negligible. Hence, $64 \%(46+18)$ of the CR get negligible citations.

\section{Letter to Editor}

Average number of letters published per year is 100 which is $17.4 \%$ of the total papers published in that year. These are able to get $9 \%$ of the total citations in that year with and average of 95. Approximately 59\% of the letters published do not get any citations at all with $17 \%$ getting only one citation which is negligible. Hence, $76 \%(59+17)$ of the letters get negligible citations.

\section{Miscellaneous}

Average 20 papers per year are published in this category. This is an average of $3.2 \%$ of the total papers published in that year. These papers get 31 citations per year which is $3.2 \%$ of the total citations received in that year. Average $49 \%$ of these papers per year do not get any citations and $14 \%$ per year get only one citation. Hence, 63\% $(49+14)$ of these papers get negligible citations.

The entire publication numbers against the percentage of papers with negligible citations is presented in the histogram (Fig. 4).

\section{h-index}

h-index, the definition of the index has been described previously it is the measure of how well the publications have been cited. Figure 5 shows the average h-index values of various categories of papers over the period of 5 years. It is evident from the graph that original articles and review articles have been able to attain higher values due to the higher citations.

The Tables 1 to 4 also show maximum citation value attained by a particular publication in a year. The values reflect the degree of academic popularity attained by the respective publication.

\section{DISCUSSION}

Judging an institutes research standing is vital for administration and for funding logistics. There are various methods to look at the performance of an institute. Citationbased analysis is one of the qualitative methods of looking at the research output. Calculation of the number of citations collected over a period of time is one of the direct evidence that the research done by that institute has served as a yardstick for other researchers. Few of the previous authors looking on the same subject have described this as intellectual influence of the scientist, institution or country ${ }^{1,5,6}$ over the subject matter. Meaning-wise citation is the reference to a published or unpublished source which usually a researcher places in his bibliographic details. In a positive sense, it is a direct acknowledgment of the quality of the cited paper and is an evidence that the research quoted has some clinical or significance in their perspectives. A good scientific paper will always be acknowledged by other continuing researches and thus will obtain greater number of citations.

The results observed after this analysis were enlightening. We observed that the OA were the top grosser of citations for both the institutes with them gathering 65 to $70 \%$ of the total citations. But still there is a significant number of $\mathrm{OA}$ which were having negligible citations (this runs close to $40 \%$ ). This kind of information points toward the quantum of insignificant or more respect fully less useful researches/publications coming from the institute. It was also observed that there is a huge bulk of minor category of publications which included case reports, letters and miscellaneous. A large bulk of this group (more than 60\%) remains uncited even after attaining adequate academic age. Although this group does forms an important part of the practice of science, the scientist must remember that these

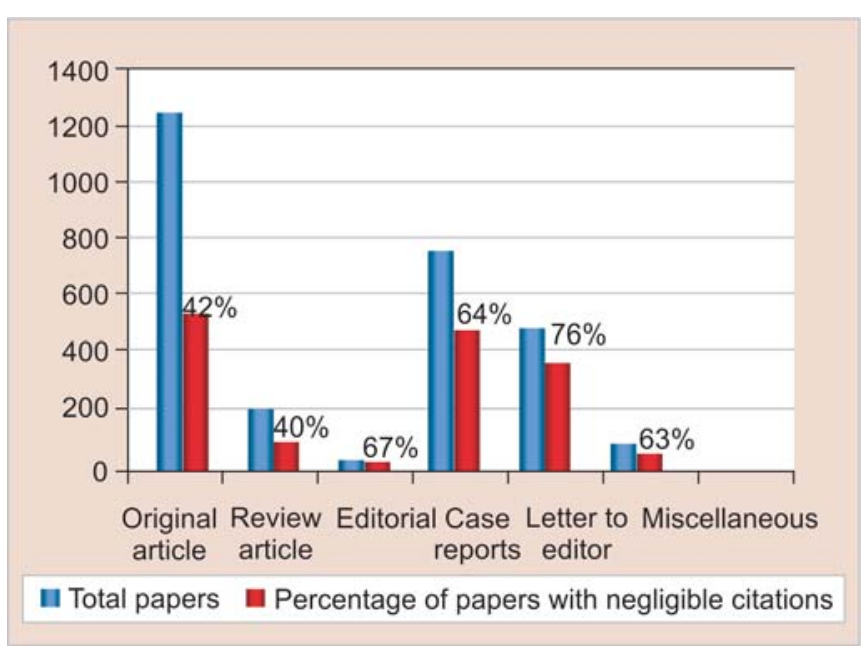

Fig. 4: Publication numbers against the percentage of papers with negligible citations from PGIMER 
publications will make a limited impact to the subject understanding.

The h-index has been explained earlier in the text, this index is a direct indicator of the quality of the research in terms of citations. It is applicable to group of documents. It serves as an alternate to traditional impact factor. The index measures the quality as well as the sustainability of the scientific output. The results of the two institutes in terms of h-index are shown in Figure 5. It was seen that higher value of h-index (18/13 for OA from AIIMS/ PGIMER respectively) is obtained with greater number of publications lying at the highest level of evidence, i.e. the randomized control trials, systemic analysis, metaanalysis, original case series (these all are grouped under $\mathrm{OA})$. Where as the other types will have poor citations and hence lower value of h-index (8/1.6 for case reports, 4/6.2 for letters, 6.2/4 for miscellaneous from AIIMS/ PGIMER respectively). There are some limitations to the h-index values which need to be mentioned. It will differ with the size of the records available with different databases, i.e. more the number of scientific periodicals indexed with a data base the higher will be the value of the h-index generated by it. Since, the number of citations achieved by a RP will increase with passage of time, i.e. the academic age of the publication. A paper even if with an important discovery will have lesser value if it has been a recent one. h-index does not consider the context of citation, so even if a paper is cited in a negative context the adding citation count will still increase the h-index, similarly self-citation will also increase the value. To overcome these limitations, several modifications and different factors were proposed but none have been proved to be ideal so far.

\section{NEED FOR THE STUDY}

We carried out this study to asses magnitude and quality of research which is being done in the mentioned institutes along with the scholarly impact of the publications in terms of citations. We also presumed that data collected in a defined manner with the analysis of observations made will serve as a benchmark for several other institutes either in India or abroad. The administration can compare their research performance by performing similar kind of analysis and their results can help them to motivate the researchers for effective research. In the past, several other authors have done a similar kind of analysis either for an individual journal, ${ }^{7}$ group of scientist ${ }^{8}$ and citation differences between two citation indexing agencies. ${ }^{9,10}$ This is the first such kind of analysis done for the two premier medical institutes in India utilizing SCA.

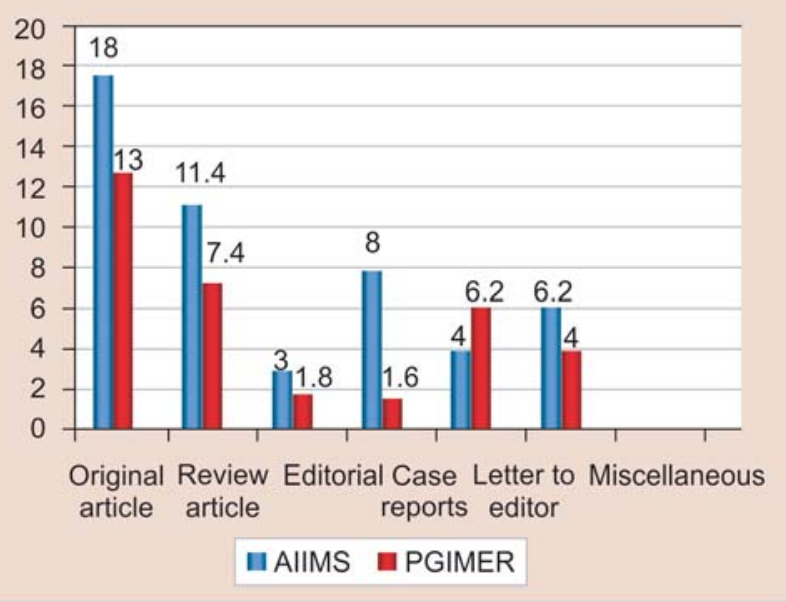

Fig. 5: Average h-index values of various categories of papers over the period of 5 years

\section{WHY SCOPUS?}

There are several online databases available which have large number of scholarly journals. Scopus, ${ }^{11}$ Web of Science, ${ }^{12}$ Science citation index, ${ }^{13}$ PubMed $^{14}$ and Google scholar, ${ }^{15,16}$ are to name a few. Of these all of them vary in terms of no of journals they index and the time duration of indexing. Scopus out of these form the largest database and indexes more than 18,000 periodicals within its system. Of these periodicals majority of the life sciences and biological sciences journals are indexed with Scopus. It is this massive data repository from Scopus coupled with the ease of search ability prompted us to do a SCA.

\section{STUDY LIMITATIONS}

The study is limited by several factors. The prime limitation being the type of indexing agency used. As previously mentioned, there are several citation databases which claim to have widest coverage of periodicals with them. The citation data will be more accurate with a database which has maximum possible periodicals listed within; conversely it will be limited by the no of the journals indexed with it. SCA was done as it had over 18,000 journal indexed with it and was accessible to us. The citation count always increases with the academic age of the publication. Hence, a relatively newer publication will have lesser counts. We can, therefore, expect that the 2008 and 2007 publication is still counting in their collection of citations. The h-index-based calculations also have inherent limitations which have been discussed above. There are other models of such kind of bibliographic assessment which have tried to overcome these shortcomings. The Becker Medical Library Model for Assessment of Research Impact is such system proposed by Washington University School of Medicine Becker Medical Library is one such method. ${ }^{17}$ They proposed that the methodology assess a RP based on five criteria's namely 
research output, knowledge transfer, clinical implementation, community benefit, legislation and policy enactment and claimed that it gives a holistic impact of the research done.

The study provides some future direction in a way that similar studies should be carried out on a five-yearly basis and the results should be compared. This will give a more accurate estimation over the quality of the work being carried out. The study findings can be seen in an international context to know our research standing. The human resources and the medical health department can take these kinds of study results as a yardstick for proportionate fund allocation in the name of research.

\section{CONCLUSION}

The quality of the research being done by either an individual or an institution should not be done by the number of RP rather a qualitative estimation should be done by the described method of citation analysis. The type of publications has an impact on the quality, the OA category of papers fetch more citations in long run hence should be seen as an indicator of good research. Close to $40 \%$ of OA get negligible citations hence the institute should focus on decreasing this group of papers. Similarly, the minor category of publications has more that $60 \%$ uncited researches. The results obtained from such study will help to set a benchmark for the studied institution as well as other institutes. This study should be periodically done to show how much we are doing or improving. They can form a basis of fund allocation in the name of research.

\section{REFERENCES}

1. Lee CS. Bibliometric analysis of the Korean Journal of Parasitology: Measured from SCI, PubMed, Scopus and Synapse databases. Korean J Parasitol 2009;47:S155-67.

2. Scopus. Available from: http://www.scopus.com/home.url [updated 2010 Jan; cited 2010 Feb 2010].

3. H-index. In Wikipedia, The Free Encyclopedia. Retrieved 14:47, February 4, 2010, from http://en.wikipedia.org/w/index.php? title=H-indexandoldid= 341862546, 4 Feb 2010.

4. Hirsch JE. An index to quantify an individual's scientific research output. PNAS 2005:102:16569-72.

5. Taubes G. Measure for measure in science. Science 1993;260: 884-86.

6. King DA. The scientific impact of nations. Nature 2004;430: 311-16.

7. Ma LS, Pan BR, Li WZ, Guo SY. Improved citation status of world journal gastroenterology in 2004: Analysis of all reference citations by WJG and citations of WJG articles by other SCI journals during 1998-2004. World J Gastroenterol 2005:7;1-6.
8. Miguel-Dasit A, Martí-Bonmatí L, Sanfeliu P. Bibliometric analysis of the Spanish MR radiological production (2001-2007). Eur J Radiol 2008;67:384-91.

9. Kulkarni AV, Aziz B, Shams I, Busse JW. Comparisons of citations in Web of Science, Scopus, and Google Scholar for articles published in general medical journals. JAMA 2009:302; 1092-96.

10. Falagas ME, Pitsouni EI, Malietzis GA, Pappas G. Comparison of PubMed, Scopus, Web of Science and Google Scholar: strengths and weaknesses. FASEB J 2008;22:338-42.

11. Elsevier. Scopus Web site. http://www.scopus.com. Accessed Dec 7, 2009

12. Web of Science. (2010, January 30). In Wikipedia, The Free Encyclopedia. Retrieved 15:24, February 4, 2010, from http:// en.wikipedia.org/w/index.php?title=Web_of_Scienceandoldid= 340977246

13. Science Citation Index. (2009, November 2). In Wikipedia, The Free Encyclopedia. Retrieved 15:23, February 4, 2010, from http://en.wikipedia.org/w/index.php?title=Science_Citation_ Indexandoldid=323406312

14. PubMed. (2010, January 29). In Wikipedia, The Free Encyclopedia. Retrieved 15:25, February 4, 2010, from http:// en.wikipedia.org/w/index.php?title=PubMedandoldid= 340641837

15. Google. Google Scholar beta Web site. http://scholar.google. com. Accessed December 7, 2009.

16. Noruzi A. Google Scholar: The new generation of citation indexes. LIBRI 2005;55:170-80.

17. Sarli CC, Dubinsky EK, Holmes KL. Beyond citation analysis: A model for assessment of research impact. J Med Libr Assoc 2010;98:17-23.

\section{ABOUT THE AUTHORS}

\section{Ravi Prakash Kanojia}

Assistant Professor, Department of Pediatric Surgery, Advanced Pediatric Center, Postgraduate Institute of Medical Education and Research, Chandigarh, India

\section{Kirti Kumar Rathod}

Senior Resident, Department of Pediatric Surgery, Advanced Pediatric Center, Postgraduate Institute of Medical Education and Research Chandigarh, India

\section{KLN Rao}

Professor and Head, Department of Pediatric Surgery, Advanced Pediatric Center, Postgraduate Institute of Medical Education and Research, Chandigarh, India

\section{CORRESPONDING AUTHOR}

Ravi Prakash Kanojia, Assistant Professor, Department of Pediatric Surgery, APC Block 3-A, Postgraduate Institute of Medical Education and Research, Sector 12, Chandigarh-160012, India e-mail: drravikanojia@yahoo.com 